\title{
microRNA-130a-5p suppresses myocardial ischemia reperfusion injury by downregulating the HMGB2/NF-KB axis
}

\author{
Yong Li ${ }^{*} \mathbb{D}$, Hongbo Zhang, Zhanhu Li, Xiaoju Yan, Yuan Li and Shuai Liu
}

\begin{abstract}
Background: Myocardial ischemia reperfusion injury (MIRI) is defined as tissue injury in the pathological process of progressive aggravation in ischemic myocardium after the occurrence of acute coronary artery occlusion. Research has documented the involvement of microRNAs (miRs) in MIRI. However, there is obscure information about the role of miR-130a-5p in MIRI. Herein, this study aims to investigate the effect of miR-130a-5p on MIRI.
\end{abstract}

Methods: MIRI mouse models were established. Then, the cardiac function and hemodynamics were detected using ultrasonography and multiconductive physiological recorder. Functional assays in miR-130a-5p were adopted to test the degrees of oxidative stress, mitochondrial functions, inflammation and apoptosis. Hematoxylin and eosin (HE) staining was performed to validate the myocardial injury in mice. Reverse transcription quantitative polymerase chain reaction (RT-qPCR) was employed to assess the expression patterns of miR-130a-5p, high mobility group box (HMGB)2 and NF-KB. Then, dual-luciferase reporter gene assay was performed to elucidate the targeting relation between miR130a-5p and HMGB2.

Results: Disrupted structural arrangement in MIRI mouse models was evident from HE staining. RT-qPCR revealed that overexpressed miR-130a-5p alleviated MIRI, MIRI-induced oxidative stress and mitochondrial disorder in the mice. Next, the targeting relation between miR-130a-5p and HMGB2 was ascertained. Overexpressed HMGB2 annulled the protective effects of miR-130a-5p in MIRI mice. Additionally, miR-130a-5p targets HMGB2 to downregulate the nuclear factor kappa-B (NF-kB) axis, mitigating the inflammatory injury induced by MIRI.

Conclusion: Our study demonstrated that miR-130a-5p suppresses MIRI by down-regulating the HMGB2/NF-KB axis. This investigation may provide novel insights for development of MIRI treatments.

Keywords: Myocardium ischemia reperfusion injury, microRNA-130a-5p, HMGB2, NF-kb pathway, Oxidative stress, Mitochondrial disorder

\section{Background}

Over production of free radicals and reactive oxygen species (ROS) has been identified as the principal cause for the pathogenesis of myocardial ischemia reperfusion injury (MIRI) [1]. Reperfusion after ischemia exacerbates the severity of ischemic injury and serves as a vital

*Correspondence: drliyong0927@163.com

Department of Cardiology, Harrision International Peace Hospital, No. 180

Renmin East Road, Hengshui 053000, Hebei, People's Republic of China cause for about a half of myocardial infarction (MI) size [2]. The cascade of various inflammatory factors induces secondary injury, exacerbating IR injury [3]. Oxidative stress consequent of MIRI in an open-heart surgery or MI aggrandizes the accumulative reactive aldehydes, thus precipitating to severe heart injury [4]. Additionally, the systematic mitochondria are an effective method to appease MIRI, accounting that the ROS-mediated mitochondrial disorder can accentuate the risk of higher susceptibility to MI, heart dysfunction and incidence of 
heart arrhythmia [5]. However, due to the lack of concrete evidence to thoroughly avoid MIRI [6], novel therapeutic strategies are imperative for the prevention of MIRI. Therefore, our study was designed to investigate the roles of different pathological mechanisms in MIRI, in order to develop novel intervention strategies.

microRNAs (miRs) are acknowledged for their involvement in MIRI by serving as modulators of pivotal signaling factors [7]. During the various MIRI states, miRs are differentially expressed to influence the MIRI biological developments [8]. Additionally, the miR-130a ectopic expression reduces ROS production in patients with oxygen-glucose deprivation reperfusion (OGDR) [9], thereby exhibiting its protective properties in patients affected with diseases associated to ischemic reperfusion. Furthermore, Lu et al. [10] have elicited that miR-130a aids in reducing MI through remodeling and promoting cardiac functions. In acute ischemic stroke tissues, miR-130a is conducive in reducing the susceptibility and severity to disease and decreasing the levels of inflammatory factors [11]. Furthermore, miR-130a-3p could accentuate the angiogenesis in $\mathrm{MI}$ as a protective measure for the ischemic heart [12]. Moreover, miR-130a-5p is downregulated in human definitive endodermal progenitor cells, which principally serves as a target for mitochondria, denoting a positive relation between miR-130a-5p and mitochondrial structure and function remodeling [13]. HMGB2 (high mobility group box 2) is regarded as a trivial factor in severe ischemic injury of MI through augmenting the inflammatory response, cell apoptosis and autophagosome clearance damage by facilitating ROS [14]. NF- $\mathrm{kB}$ serves as an integrated transcriptional factor in cardiovascular disease [15]. NF- $\mathrm{kB}$ is highly expressed in lung IRI rats [16]. HMGB2 ${ }^{-/-}$mouse cells are defective in type-I interferon and inflammatory cytokine, showing impaired activation of NF- $k B$ [17]. From the aforementioned literature, it is hypothesized that miR-130a-5p/ HMGB2/NF-кB can be a viable target in MIRI treatment. Thus, our experiments were performed to substantiate the hypothesis.

\section{Methods}

\section{Animal grouping}

A total of sixty healthy C57BL/6 male mice (6-8-weekold, 20-25 g) (Guangzhou General Pharmaceutical Research Institute Co., Ltd., Guangzhou, Guangdong, China, SYXK (Guangdong) 2018-0003) raised in standard conditions were numbered with body weight as a parameter and randomly allocated into the sham group, MIRI group, agomir negative control (NC) group, miR-130a-5p agomir group, miR-130a-5p agomir + pLVX-Puro group and miR-130a-5p agomir + pLVX-Puro-HMGB group, with 10 mice in each group. Mice in the sham group were threaded in the left anterior descending coronary artery without ligation. Mice in the MIRI group were anesthetized using an intraperitoneal injection of $4 \%$ pentobarbital sodium (40 mg/kg), after which their limbs were connected to the BL-420F physiological monitor system (Techman Science and Technology Co., Ltd., Chengdu, Sichuan, China) for subsequent electrocardiography, and their chests were dissected on the fourth rib, and ligated for $30 \mathrm{~min}$ via the left cardiac anterior descending coronary artery and then underwent a 120-min reperfusion. Correspondingly, the mice in the remaining 4 groups $24 \mathrm{~h}$ before MIR were subjected to an intramyocardial injection of agomir NC (Guangzhou RiboBio Co., Ltd, Guangzhou, Guangdong China), miR-130a-5p agomir $(50 \mu \mathrm{L}, 300 \mathrm{nmol} / \mathrm{kg})$ [18] (RiboBio), miR-130a-5p agomir + pLVX-Puro $\left(1 \times 10^{7} \mathrm{pfu}\right)$ (Gaining Biotechnology Co., Ltd., Shanghai, China) and miR-130a-5p agomir + pLVX-Puro-HMGB2 $\left(1 \times 10^{7} \mathrm{pfu}\right)$ (Gaining) respectively.

\section{Detection of cardiac function and hemodynamics in mice} After reperfusion, the Philips Sonos 5500 color Doppler ultrasonography (Philips, Andover, MA, USA) was utilized to determine the left ventricle (LV) ejection fraction (EF) and LV fraction shortening (FS). After the echocardiography, numerous self-made catheters with heparin saline were inserted via the right common carotid artery of mice into the LV. Then, the systolic pressure (SP), enddiastolic pressure (EDP) and the maximal rate of rise and fall $( \pm \mathrm{dp} / \mathrm{dtmax})$ of the LV were analyzed and documented using a multi-conductive physiological recorder (Chengdu Medical Instruments Co., Ltd., Chengdu, Sichuan, China).

\section{Sample collection}

After the hemodynamics detection, the mice were euthanized using an intraperitoneal injection with $40 \mathrm{mg} / \mathrm{kg}$ pentobarbital sodium, and $1 \mathrm{~mL}$ blood samples collected from each mouse by cardiac puncture were placed for $10 \mathrm{~min}$ and then centrifuged at $3000 \mathrm{~g}$ for $10 \mathrm{~min}$. The $200 \mu \mathrm{L}$ serum was extracted and preserved at $-80^{\circ} \mathrm{C}$ for subsequent experimentation. After collecting the blood samples, the mice were sacrificed by an intraperitoneal injection of excessive pentobarbital sodium, after which the myocardial tissues were harvested. Among each group, 4 samples of myocardial tissues were adopted to prepare tissue homogenate and the remaining 6 samples were fixed using $4 \%$ paraformaldehyde for $24 \mathrm{~h}$ and were methodically prepared into paraffin-sections for histological staining. 


\section{Enzyme-linked immunosorbent assay (ELISA)}

The serum was diluted, and the levels of creatine kinase (CK), T Cardiac troponin $\mathrm{T}$ (cTnT), lactate dehydrogenase (LDH), superoxide dismutase (SOD) and malondialdehyde (MDA) in the mouse serum $(20 \mu \mathrm{L} /$ well $)$ were analyzed in duplicates in strict accordance with the provided instructions (NanJing JianCheng Bioengineering Institute, Nanjing, Jiangsu, China). The levels of tumor necrosis factor- $\alpha$ (TNF- $\alpha$ ), interleukin (IL)- 6 and IL-1 $\beta$ were evaluated using the enzyme-linked immunosorbent assay kits (Shanghai Enzyme-linked Biotechnology Co., Ltd., Shanghai, China).

\section{Measurement of mitochondrial functions}

With the addition of the mitochondrial separation reagent (Beyotime Biotechnology Co., Ltd., Shanghai, China), the tissue homogenate was centrifuged at $600 \mathrm{~g}$ at $4{ }^{\circ} \mathrm{C}$ for $5 \mathrm{~min}$, and the supernatant was extracted and centrifuged at $1100 \mathrm{~g}$ at $4{ }^{\circ} \mathrm{C}$ for $10 \mathrm{~min}$, followed by supernatant removal. Next, the mitochondria were resuspended using the mitochondrial storage fluid for the detection of various mitochondrial function-related indices. JC-1 kit (Beyotime) was employed to assess the mitochondria membrane potential (MMP). Additionally, the levels of adenosine triphosphate (ATP), ROS and calcium ion were measured based on the provided instructions of the ATP kit (Shanghai Enzyme-linked Biotechnology), ROS kit (Shanghai Enzyme-linked Biotechnology) and the calcium ion detection kit (Sigma-Aldrich, Merck KGaA, Darmstadt, Germany) respectively.

\section{Hematoxylin and eosin (HE) staining}

The regularly dewaxed and dehydrated paraffin-sections were stained using hematoxylin (Beijing Solarbio Science and Technology Co., Ltd., Beijing, China) for $3 \mathrm{~min}$ and then rinsed. Subsequently, these sections were differentiated using $1 \%$ hydrochloric alcohol for $15 \mathrm{~s}$. After a regimen of 2-min staining using eosin (Solarbio), the sections were observed under an optical microscope (Olympus Optical Co., Ltd., Tokyo, Japan).

\section{Terminal deoxynucleotidyl transferase (TdT)-mediated dUTP nick-end labeling (TUNEL) staining}

After the paraffin-sections were regularly dewaxed and dehydrated, they were analyzed in strict accordance with the provided instructions of the TUNEL apoptosis detection kit (Shanghai Yeason Bio Technologies Co., Ltd., Shanghai, China). Next, the nuclei were stained with 4',6-diamidino-2-phenylindole (Beyotime) for observation of the TUNEL-positive cells under a fluorescence microscope (Olympus).

\section{Reverse transcription-quantitative polymerase chain reaction (RT-qPCR)}

The total RNA content from the tissue homogenate of each group was extracted using a TRIzol kit (Beyotime) for assessment of RNA concentration and purity. Then, the extracted RNA content was reverse transcribed into cDNA using the Rever Tra Ace qPCR RT Master Mix kit (Toyobo Co., Ltd., Tokyo, Japan). SYBR Premix Ex Taq ${ }^{\text {TM }}$ II (Takara, Dalian, China) was applied to perform fluorescent qPCR. The $2^{-\Delta \Delta C t}$ method was adopted to calculate the relative expression of the genes with glyceraldehyde3-phosphate dehydrogenase (GAPDH) as the internal reference (Table 1).

\section{Western blot analysis}

The total protein content from the tissue homogenate of each group was extracted for measurement of protein concentration. Next, the protein content was subjected to sodium dodecyl sulfate polyacrylamide gel electrophoresis (Beyotime) and then transferred onto polyvinylidene fluoride membranes. Then, the membranes were sealed using 5\% skim milk powder for $1 \mathrm{~h}$ and cultured with the following primary antibodies (all from Abcam Inc., Cambridge, MA, USA): B-cell lymphoma-2 (Bcl-2) (ab59348, 1:1000), Bcl2-associated X (Bax) (ab182733, 1:2000), Cytochrome C (Cyt-c) (ab216971, 1:1000), apoptosis inducing factor (AIF) (ab32516, 1:1000), HMGB2 (ab124670, 1:10,000), p65 (ab16502, $0.5 \mu \mathrm{g} / \mathrm{mL}$ ), inhibitor of $\kappa B$ (IкB) $\alpha$ (ab109300, 1:1000), p-p65 (ab86299, 1:2000) and $\mathrm{p}-\mathrm{I} \kappa \mathrm{B} \alpha(\mathrm{ab} 133478,1: 10,000)$ at $4{ }^{\circ} \mathrm{C}$ overnight. Next, the membranes were cultivated using the horseradish peroxidase labeled goat anti-rabbit immunoglobulin G (IgG) antibody (ab32152, 1:2000) or the anti-mouse IgG antibody (ab205719, 1:2000) at room temperature for $1 \mathrm{~h}$ and then exposed for visualization and observation.

\section{Table 1 Primers sequence}

\begin{tabular}{ll}
\hline Gene & Primers $\left(\mathbf{5}^{\prime}-\mathbf{3}^{\prime}\right)$ \\
\hline miR-130a-5p & $\begin{array}{l}\text { Forward: 5'-CCAGGGCTTTTCAAAAATGA-3' } \\
\text { U6 }\end{array}$ \\
& Reverse: 5'-CCGATCCAATCTGTTCTGGT-3' \\
& Forward primer: 5'-GTGCTCGCTTCGGCAGCA-3' \\
HMGB2 & Feverse primer: 5'-CAAAATATGGAACGCTTC-3' \\
& Reverse primer: 5'-AAACAGGAAGAAGGCAGATGG-3' \\
GAPDH & Forward primer: 5'-GTCAACGGATTTGGTCTGTATT-3' \\
& Reverse primer:5'-AGTCTTCTGGGTGGCAGTGAT-3' \\
\hline
\end{tabular}

miR microRNA, HMGB high mobility group protein, GAPDH glyceraldehyde-3phosphate dehydrogenase 
GAPDH (ab8245, 1:1000) served as the internal reference, and the ratio of the gray value of the target protein band to GAPDH was used as the relative protein expression.

\section{Dual-luciferase reporter gene assay}

TargetScan predicted the presence of numerous binding sites between miR-130a-5p and HMGB2. The complementary binding sequence of miR-130a-5p and HMGB2 was amplified using PCR and cloned to the pmiR-GLO luciferase vector (Promega, Madison, WI, USA) in order to construct HMGB2 wild type (WT) plasmids and HMGB2 mutant type (MUT) plasmids. Then, the two kinds of plasmids were combined with mimic NC and miR-130a-5p mimic and then transfected into the 293 T cells with Lipofectamine ${ }^{\mathrm{TM}} 2000$ (Invitrogen Inc., Carlsbad, CA, USA). The luciferase activity was evaluated $48 \mathrm{~h}$ later. All experiments were conducted 3 times independently.

\section{Statistical analysis}

SPSS 21.0 (IBM Corp. Armonk, NY, USA) was employed for statistical data analysis. Kolmogorov-Smirnov test was employed to assess whether the data were in normal distribution. The results were shown in mean \pm standard deviation. One-way analysis of variance (ANOVA) or two-way ANOVA was adopted for comparing among different groups, and the Tukey's multiple comparisons test was adopted for pairwise comparisons after ANOVA. The $p$ value was attained using a two-tailed test, where a value of $p<0.05$ was indicative of a significant difference.

\section{Results}

\section{Identification of MIRI mouse models}

In comparison with the sham group, the ST segment of mice in the MIRI group rose and peaked until $30 \mathrm{~min}$ after ischemia and regressed at the 120 min after reperfusion (Fig. 1a). Results of HE staining observed consistent cardiomyocyte structure with orderly and tight myocardial fiber arrangement in the sham-operated mice; while in the MIRI mice, the broken and light-stained myocardial fibers were disorderly arranged with an extending interim between each other (Fig. 2b).

\section{Overexpressed miR-130a-5p alleviates MIRI in mice}

In comparison with the sham-operated mice, the MIRI mice presented with a lower miR-130a-5p expression pattern, which was increased after overexpressing miR130a-5p (all $p<0.01$ ) (Fig. 2a). In the MIRI group, the EF, FS, LVSP and $\pm d p / d t m a x$ were all reduced and LVEDP was elevated; while overexpressed miR-130a-5p resulted in contradictory outcomes relative to those in the sham group (all $p<0.01$ ) (Fig. 2b, c). Significant increases were observed in the CK and cTnT contents in serum from the MIRI mice, which were repressed by overexpressed miR-130a-5p (all $p<0.01$ ) (Fig. 2d). According to the HE staining, MIRI in mice was alleviated with miR-130a-5p overexpression (Fig. 2e). TUNEL assay revealed a high apoptosis rate in the cardiomyocytes in MIRI mice, which was lowered by overexpressing miR-130a-5p (all $p<0.01)$ (Fig. 2f). Western blot analysis revealed that the increase of Bax expression and decrease of Bcl- 2 expression in the mice under MIRI were reversed by miR-130a-5p overexpression (all $p<0.01$ ) (Fig. 2g).

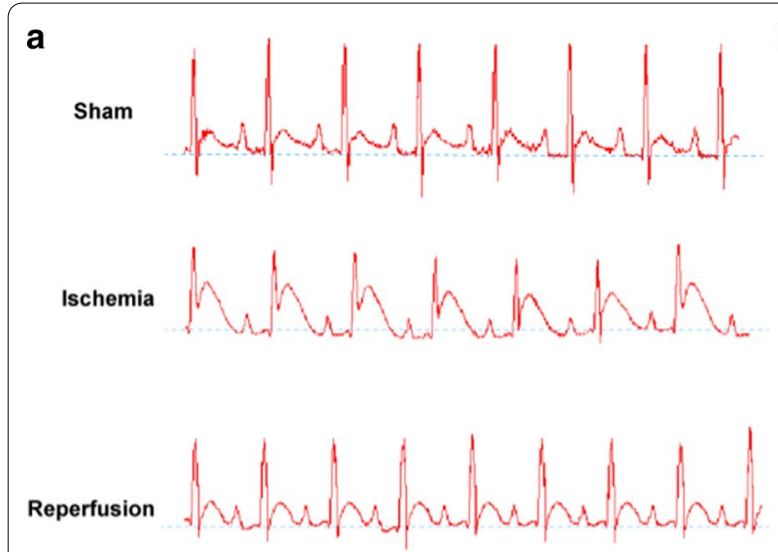

b

Fig. 1 Identification of MIRI mouse models. a The electrocardiography of the MIRI mice, ischemia: 30 min, reperfusion: 120 min, $n=10$. $\mathbf{b}$ HE staining revealed that the myocardial tissues were disrupted in the MIRI mice, $\times 200, n=6$. MIRI myocardial ischemia reperfusion injury, HE hematoxylin and eosin 


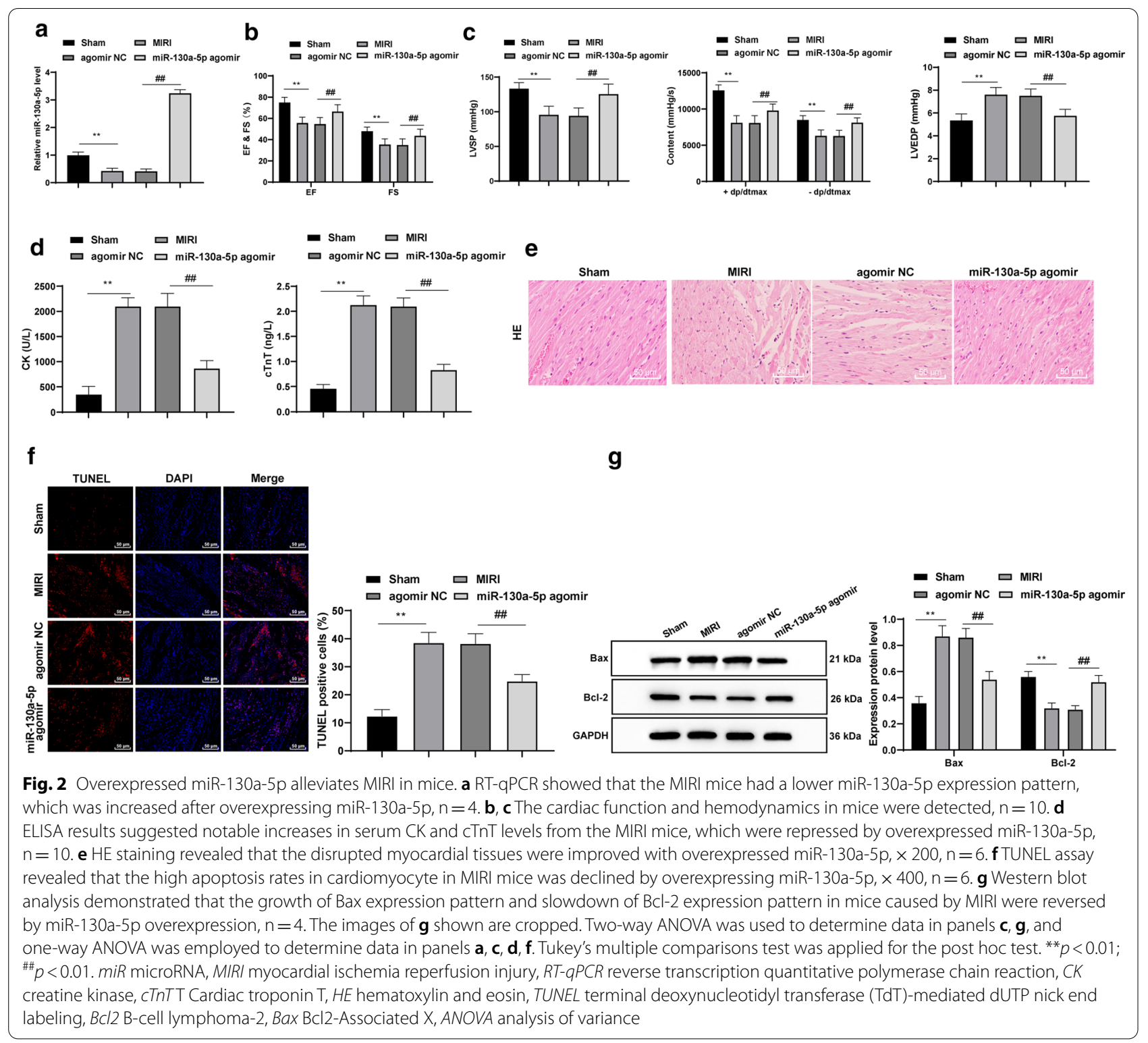

Overexpressed miR-130a-5p alleviates MIRI-induced oxidative stress and mitochondrial disorder in mice

Relative to those in the sham group, the decreases in SOD, MMP and ATP as well as increases in LDH, MDA, ROS, $\mathrm{Ca}^{2+}$, Cyt-c and AIF protein levels were evident in the MIRI group, which were inverted by overexpressed miR-130a-5p (all $p<0.01$ ) (Fig. 3a-c).

\section{miR-130a-5p targets HMGB2}

TargetScan identified the binding site between miR130a-5p and HMGB2 $3^{\prime}$ untranslated region (Fig. 4a). According to the results from the dual-luciferase reporter gene assay, in comparison with the control group, miR130a-5p mimic significantly reduced the HMGB2-WT luciferase activity (both $p<0.01$ ) (Fig. 4b). Additionally, the mRNA expression and protein level of HMGB2 in the MIRI mice were lowered by overexpressed miR-130a-5p (all $p<0.01$ ) (Fig. 4c, d).

\section{Overexpressed HMGB2 reverses the protective effects of miR-130a-5p on MIRI mice}

In comparison with the miR-130a-5p agomir group, when HMGB2 was upregulated in the context of miR130a-5p overexpression, the mRNA expression and protein level of HMGB2 were evidently increased (all $p<0.01$ ) (Fig. 5a, b); EF, FS, LVSP and $\pm \mathrm{dp} / \mathrm{dtmax}$ were lowered while the LVEDP was enhanced (all $p<0.01$ ) (Fig. 5c, d); CK and cTnT levels in the serum were elevate 


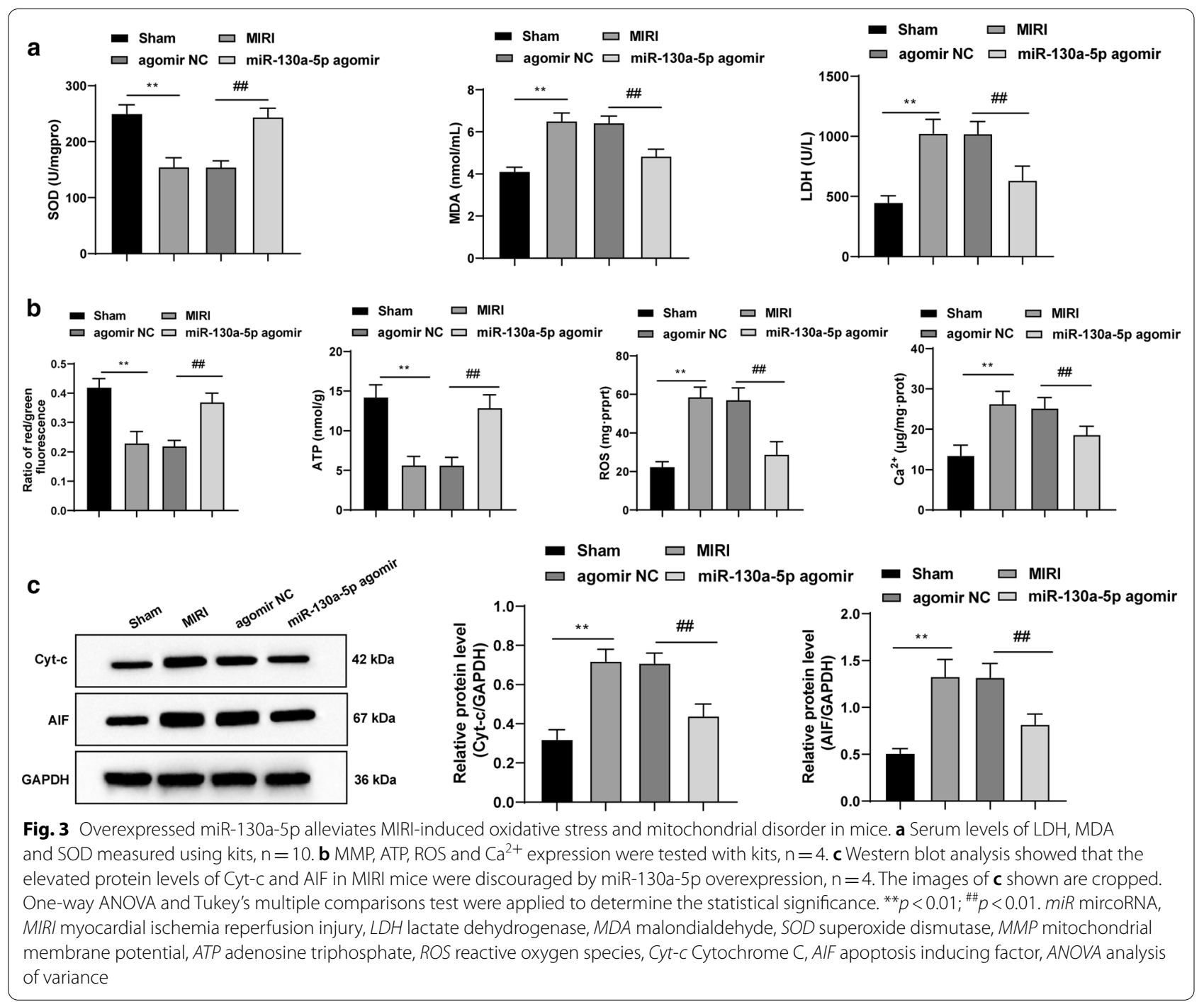

(all $p<0.01)$ (Fig. 5e), myocardial injury was further deteriorated (Fig. $5 \mathrm{f}$ ), the apoptosis rate of cardiomyocytes were enhanced (all $p<0.01$ ) (Fig. 5g), Bax expression was elevated and Bcl-2 expression was reduced (all $p<0.01$ ) (Fig. 5h).

miR-130a-5p targets HMGB2 to downregulate the NF-KB axis, mitigating inflammatory injury induced by MIRI In comparison with the sham-operated mice, in the MIRI mice, p65 and $\mathrm{I} \mathrm{kB} \alpha$ phosphorylation in the myocardial tissues was enhanced along with elevated serum levels of TNF- $\alpha$, IL- 6 and IL- $1 \beta$. Overexpressing miR-130a-5p devoted to inverted results. However, upon up-regulation of HMGB2 in the MIRI mice with overexpressed miR$130 \mathrm{a}-5 \mathrm{p}, \mathrm{p} 65$ and IkB $\alpha$ phosphorylation in the myocardial tissues was enhanced and the serum levels of TNF- $\alpha$,
IL-6 and IL-1 $\beta$ were also elevated correspondingly (all $p<0.01$ ) (Fig. 6a, b).

\section{Discussion}

The miR-130a expression was lower in the OGDRtreated cells compared to the normoxic cells [9]. Among the vast medical literature about MIRI, however, theme related to miR-130a-5p was seldom found, so we aimed to elucidate some potential MIRI therapies on the basis of miR-130a-5p. Consequently, our data showed that overexpressed miR-130a-5p suppressed MIRI by downregulating the HMGB2/NF- $\mathrm{kB}$ axis.

Firstly, our results demonstrated significant decreases of EF, FS, LVSP and $\pm d p / d t m a x$ along with a notable increase of LVEDP. As a diagnostic index of MIRI injury, decreased LVDP and $\pm d p / d t m a x$ inferred a wide range of MI [19]. Then, our findings exhibited elevated CK and cTnT levels in the MIRI mice. As a pivotal diagnostic 


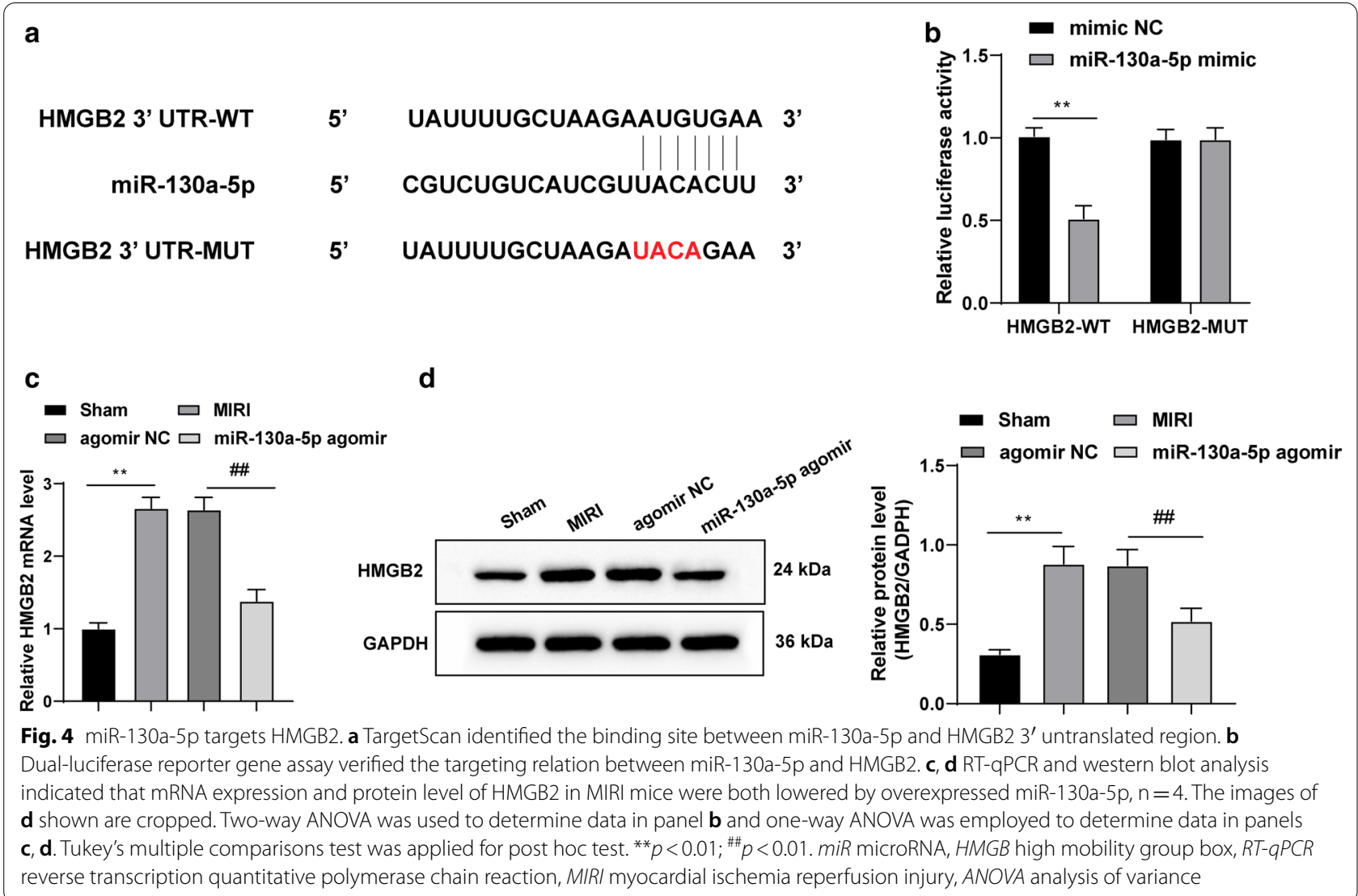

biomarker in MIRI, the activation of cTnT could have a detrimental impact on heart function [20]. CK and cTnT as common targets in heart disease were correlated with several cardiac specific miRs for regulating acute myocardial ischemia [21]. Consistently, these findings served as basis for a speculation supporting that miR-130-5p could downregulate $\mathrm{CK}$ and $\mathrm{cTnT}$ to subsequently alleviate MIRI progression. Also, MIRI mice presented with a high apoptosis rate, where Bax was up-regulated while $\mathrm{Bcl}-2$ was down-regulated. Lower Bcl-2 level and higher Bax level were correspondingly evident in renal tissue subjected to IRI relative to the normal renal tissues [22]. In cerebral IRI, miR-130a hindered cell apoptosis induced by OGDR [9]. Subsequent gain-of assays suggested that overexpressed miR-130a-5p ameliorated MIRI by contributing to an impeded cardiomyocyte apoptosis and repairing the MIRI structural arrangement. Additionally, miR-130a, could help cells maintain homeostasis under hypoxic conditions induced due to oxidative stress, therefore alleviating peripheral artery disease [23]. Besides, overexpressed miR-130a-5p contributed to ameliorate oxidative stress and mitochondrial disorder consequent of MIRI. Additionally, miRs extensively modulated the mitochondrial activities, the chief organelle for cell apoptosis after MIRI [7]. miR-130a exerted protective effects against cerebral IRI [11]. The regulation of miR-130a-3p on mitochondrial functions was a potential development in fighting against cell senescence [24]. The aforementioned results discernibly indicated that miR130a-5p relieved MIRI to a significant degree.

A prior study identified the oncogene HMGB2 as a downstream target of miR-130a [25]. Additionally, the results from dual-luciferase reporter gene assay ascertained the targeting relation between miR-130a-5p and HMGB2. In the glioma cells, miR130a-5p overexpression exercised repressive effects by targeting HMGB2 [26]. Furthermore, our findings demonstrated that overexpressed HMGB2 could avert the protective effects of miR-130a-5p on MIRI mice. After IR, HMGB2 was elevated in the cardiomyocytes after MIRI, which enhanced the cell apoptosis, and led to increased Bax and decreased Bcl-2 levels [27]. Increased serum HMGB2 levels were associated with MI severity and deleterious cardiac events; hence the increased HMGB2 levels amplified myocardial ischemic injury in rats and hypoxic $\mathrm{H} 9 \mathrm{C} 2$ cell damage via ROS induced by progressive glycation end products [28]. In hepatic IR, where HMBG1 was activated, 


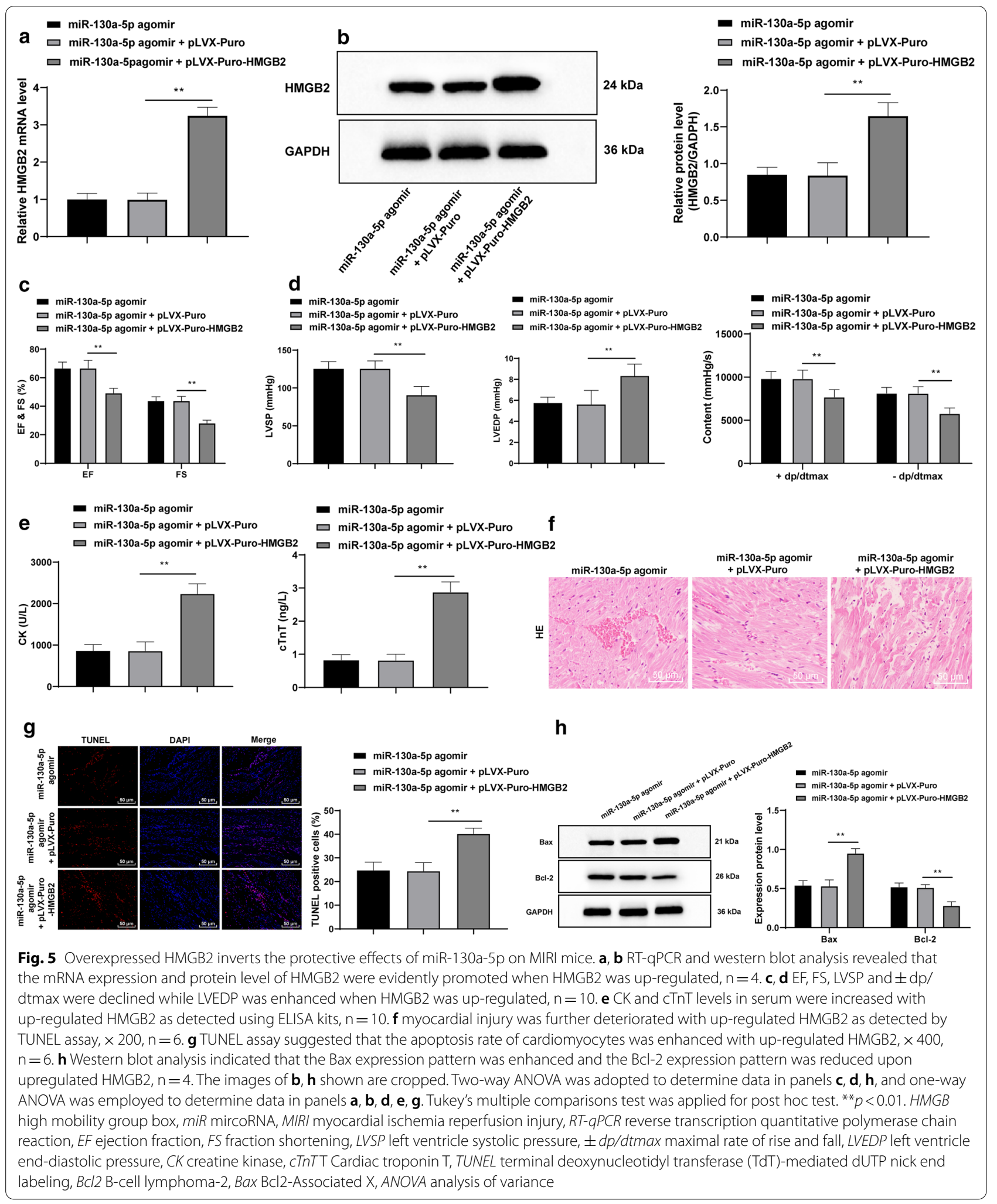

the MDA level was also enhanced, conferring to a positive association between HMGB and oxidative stress
[14]. As the major source of ROS and a vital mediator in the apoptotic process, mitochondria combined with 


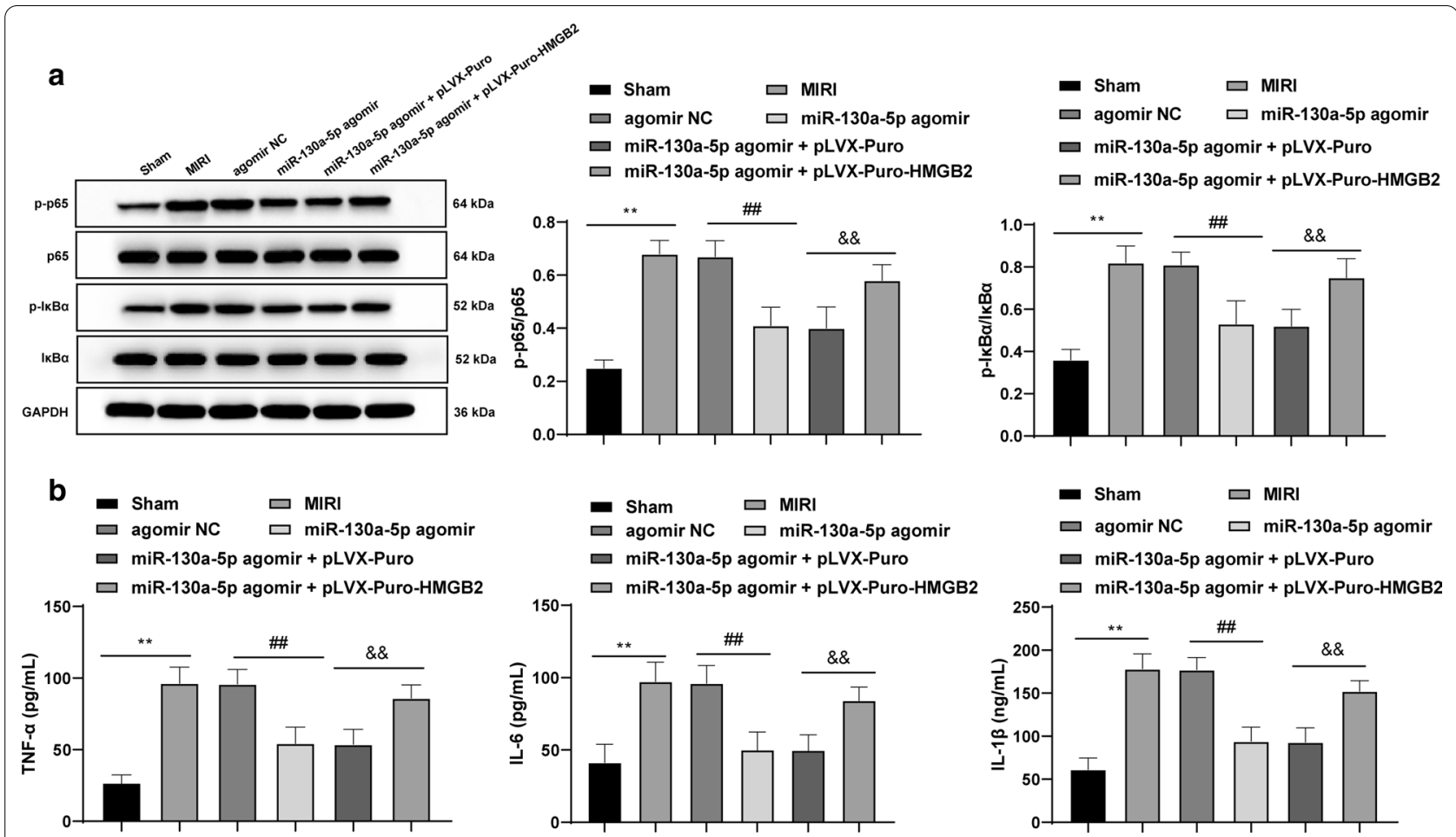

Fig. 6 miR-130a-5p targets HMGB2 to downregulate the NF-KB axis, mitigating inflammatory injury induced by MIRI. a Western blot analysis was applied to assess the p65 and IKBa protein levels and phosphorylation in myocardial tissues in MIRI mice, $n=4$. $\mathbf{b}$ ELISA kits were utilized to determine the serum levels of TNF- $\mathrm{a}, \mathrm{IL}-6$ and IL-1 $\beta, n=10$. The images of a shown are cropped. One-way ANOVA and Tukey's multiple comparisons test were applied to determine statistical significance. ${ }^{* *} p<0.01 ; \# p<0.01 ;{ }^{\& \&} p<0.01$. miR microRNA, HMGB high mobility group box, NF-KB nuclear factor kappa-B, MIRI myocardial ischemia reperfusion injury, IKB inhibitor of $\mathrm{KB}$, ELISA enzyme-linked immunosorbent assay, TNF- $a$ tumor necrosis factor-a, $1 L$ interleukin

the inflammation-triggered HMBG1to further exacerbate IRI [29]. Moreover, our findings revealed that miR-130a-5p targeted HMGB2 to downregulate the $\mathrm{NF}-\mathrm{KB}$ axis so as to mitigate the inflammatory injury induced by MIRI. The involvement of NF-kB axis has been in reported in hepatic IR injury and renal $I / R$ injury $[15,30]$. HMGB2 silencing significantly inhibited I/R-induced cell proliferation reduction, cell apoptosis, activation of NF-kBp65 [16]. HMGB1 also aggravated myocardial $I / R$ injury via regulation of the JNK $1 / 2$ and NF-kB pathway [31]. Captivatingly, slowdown of HMGB2 resulted in a reduced expression pattern of NF-KB in the cardiomyocytes under MIRI, which inferred the positive correlation between HMGB2 and NF- $\mathrm{kB}$ [27]. In ischemic stroke, manifestation of downregulated NF- $\mathrm{kB}$ was supplemented by the knockdown of TNF- $\alpha$, IL- 6 and IL-1 $\beta$ [32]. A recent study suggested that miR-130a targeted several pro-inflammatory cytokines to reduce the production of TNF- $\alpha$, IL- 6 and IL-12 [33]. All in all, miR-130a-5p was an attractive biomarker for relieving MIRI.

\section{Conclusion}

To conclude, our study supported that overexpressed miR-130a-5p alleviated MIRI by downregulating the HMGB2/NF- $\kappa B$ axis. These results elicited a novel approach for the development of new MIRI treatment. Our future studies will focus at exploring the underlying mechanism of other targets of miR-130a-5p and to identify reliable therapeutic targets for MIRI. As this is a preclinical research, our findings provide therapeutic implication in MIRI treatment, and however our experiment results and effective application into clinical practice need further validation.

\section{Abbreviations}

AIF: Apoptosis inducing factor; ANOVA: Analysis of variance; ATP: Adenosine triphosphate; Bax: Bcl2-Associated X; CK: Creatine kinase; CTnT: T Cardiac troponin T; Cyt-c: Cytochrome $C_{;} \pm d p / d t m a x:$ Maximal rate of rise and fall; EDP: End-diastolic pressure; EF: Ejection fraction; FS: Fraction shortening; GAPDH: Glyceraldehyde-3-phosphate dehydrogenase; HE: Hematoxylin and eosin; HMGB: High mobility group box; IL: Interleukin; IKB: Inhibitor of Kb; LDH: Lactate dehydrogenase; LV: Left ventricle; MDA: Malondialdehyde; MI: Myocardial infarction; miR: MircoRNA; MIRI: Myocardial ischemia reperfusion injury; MUT: Mutant type; NC: Negative control; NF-KB: Nuclear factor kappa-B; OGDR: Oxygen-glucose deprivation reperfusion; ROS: Reactive oxygen species; RT-qPCR: Reverse transcription quantitative polymerase chain reaction; SOD: 
Superoxide dismutase; SP: Systolic pressure; TNF-a: Tumor necrosis factor-a; TUNEL: TdT-mediated dUTP nick-end labeling; WT: Wild type.

\section{Acknowledgements}

Not applicable.

\section{Authors' contributions}

$\mathrm{YL}$ is the guarantor of integrity of the entire study and contributed to the concepts and design of this study; HBZ contributed to the definition of intellectual content and literature research of this study; $Y \mathrm{~L}$ and $\mathrm{ZHL}$ contributed to the experimental studies; XJY and ZHL contributed to the data acquisition and analysis; $Y L$ and SL took charge of the manuscript preparation; $Y L$ and HBZ wrote the manuscript. All authors read and approved the final manuscript.

\section{Funding}

Not applicable.

\section{Availability of data and materials}

The datasets used and/or analysed during the current study available from the corresponding author on reasonable request.

\section{Ethics approval and consent to participate}

This study was approved and supervised by the ethics committee of Harrision International Peace Hospital. Significant efforts were made in order to minimize both the number of animals used as well as their respective suffering.

\section{Consent for publication}

Not applicable.

\section{Competing interests}

The authors declare that they have no competing interests.

Received: 30 December 2019 Accepted: 13 October 2020 Published online: 03 March 2021

\section{References}

1. Halladin NL. Oxidative and inflammatory biomarkers of ischemia and reperfusion injuries. Dan Med J. 2015;62(4):B5054.

2. Ndrepepa G, Colleran R, Kastrati A. Reperfusion injury in ST-segment elevation myocardial infarction: the final frontier. Coron Artery Dis. 2017;28(3):253-62.

3. Shi J, Dai W, Kloner RA. Therapeutic hypothermia reduces the inflammatory response following ischemia/reperfusion injury in rat hearts. Ther Hypothermia Temp Manag. 2017;7(3):162-70.

4. Ding J, Yang Z, Ma H, Zhang H. Mitochondrial aldehyde dehydrogenase in myocardial ischemic and ischemia-reperfusion injury. Adv Exp Med Biol. 2019;1193:107-20.

5. Thummasorn S, Apaijai N, Kerdphoo S, Shinlapawittayatorn K, Chattipakorn SC, Chattipakorn N. Humanin exerts cardioprotection against cardiac ischemia/reperfusion injury through attenuation of mitochondrial dysfunction. Cardiovasc Ther. 2016;34(6):404-14.

6. Mokhtari-Zaer A, Marefati N, Atkin SL, Butler AE, Sahebkar A. The protective role of curcumin in myocardial ischemia-reperfusion injury. J Cell Physiol. 2018;234(1):214-22.

7. Makhdoumi P, Roohbakhsh A, Karimi G. MicroRNAs regulate mitochondrial apoptotic pathway in myocardial ischemia-reperfusion-injury. Biomed Pharmacother. 2016:84:1635-44.

8. Siebert $V$, Allencherril J, Ye Y, Wehrens XHT, Birnbaum Y. The role of noncoding RNAs in ischemic myocardial reperfusion injury. Cardiovasc Drugs Ther. 2019;33(4):489-98.

9. Zheng T, Shi Y, Zhang J, Peng J, Zhang X, Chen K, et al. MiR-130a exerts neuroprotective effects against ischemic stroke through PTEN/PI3K/AKT pathway. Biomed Pharmacother. 2019;117:109117.

10. Lu C, Wang X, Ha T, Hu Y, Liu L, Zhang X, et al. Attenuation of cardiac dysfunction and remodeling of myocardial infarction by microRNA-130a are mediated by suppression of PTEN and activation of PI3K dependent signaling. J Mol Cell Cardiol. 2015;89(Pt A):87-97.
11. Jin F, Xing J. Circulating miR-126 and miR-130a levels correlate with lower disease risk, disease severity, and reduced inflammatory cytokine levels in acute ischemic stroke patients. Neurol Sci. 2018;39(10):1757-65.

12. Moghaddam AS, Afshari JT, Esmaeili SA, Saburi E, Joneidi Z, MomtaziBorojeni AA. Cardioprotective microRNAs: lessons from stem cell-derived exosomal microRNAs to treat cardiovascular disease. Atherosclerosis. 2019;285:1-9.

13. Ma Y, Ma M, Sun J, Li W, Li Y, Guo X, et al. CHIR-99021 regulates mitochondrial remodelling via beta-catenin signalling and miRNA expression during endodermal differentiation. J Cell Sci. 2019;132(15):jcs229948.

14. Sherif IO, Al-Shaalan NH. Vildagliptin attenuates hepatic ischemia/reperfusion injury via the TLR4/NF-kappaB signaling pathway. Oxidative Med Cell Longev. 2018;2018:3509091.

15. Yang X, Li C, Ng KT, Liu J, Liu H, Zhang W, Xiao F, Li X, Lo CM, Lu L, Man K. IL-17a exacerbates hepatic ischemia-reperfusion injury in fatty liver by promoting neutrophil infiltration and mitochondria-driven apoptosis. J Leukoc Biol. 2020. https://doi.org/10.1002/JLB.3MA0520-716R.

16. Zhang W, Zhang Y, Ding K, Zhang H, Zhao Q, Liu Z, Xu Y. Involvement of JNK1/2-NF-kappaBp65 in the regulation of HMGB2 in myocardial ischemia/reperfusion-induced apoptosis in human AC16 cardiomyocytes. Biomed Pharmacother. 2018;106:1063-71.

17. Yanai H, Ban T, Wang Z, Choi MK, Kawamura T, Negishi H, Nakasato M, Lu Y, Hangai S, Koshiba R, Savitsky D, Ronfani L, Akira S, Bianchi ME, Honda K, Tamura T, Kodama T, Taniguchi T. HMGB proteins function as universal sentinels for nucleic-acid-mediated innate immune responses. Nature. 2009;462(7269):99-103.

18. Zhao Z, Qu F, Liu R, Xia Y. Differential expression of miR-142-3p protects cardiomyocytes from myocardial ischemia-reperfusion via TLR4/NFkB axis. J Cell Biochem. 2019. https://doi.org/10.1002/jcb.29506.

19. Zhang WY, Zhang QL, Xu MJ. Effects of propofol on myocardial ischemia reperfusion injury through inhibiting the JAK/STAT pathway. Eur Rev Med Pharmacol Sci. 2019;23(14):6339-45.

20. Wang Y, Qi X, Wang C, Zhao D, Wang H, Zhang J. Effects of propofol on myocardial ischemia-reperfusion injury in rats with type-2 diabetes mellitus. Biomed Rep. 2017;6(1):69-74.

21. Mushtaque RS, Hameed S, Mushtaque R, Idrees M, Siraj F. Role of cardiospecific micro-ribonucleic acids and correlation with cardiac biomarkers in acute coronary syndrome: a comprehensive systematic review. Cureus. 2019;11(10):e5878.

22. Shen S, Zhou J, Meng S, Wu J, Ma J, Zhu C, et al. The protective effects of ischemic preconditioning on rats with renal ischemia-reperfusion injury and the effects on the expression of $\mathrm{BCl}-2$ and Bax. Exp Ther Med. 2017;14(5):4077-82.

23. Signorelli SS, Volsi GL, Pitruzzella A, Fiore V, Mangiafico M, Vanella L, et al. Circulating miR-130a, miR-27b, and miR-210 in patients with peripheral artery disease and their potential relationship with oxidative stress. Angiology. 2016;67(10):945-50.

24. Giuliani A, Prattichizzo F, Micolucci L, Ceriello A, Procopio AD, Rippo MR. Mitochondrial (Dys) function in inflammaging: do MitomiRs influence the energetic, oxidative, and inflammatory status of senescent cells? Mediat Inflamm. 2017;2017:2309034.

25. Tang C, Yang Z, Chen D, Xie Q, Peng T, Wu J, Qi S. Downregulation of miR130a promotes cell growth and epithelial to mesenchymal transition by activating HMGB2 in glioma. Int J Biochem Cell Biol. 2017;93:25-31.

26. Xu CH, Xiao LM, Liu Y, Chen LK, Zheng SY, Zeng EM, et al. The IncRNA HOXA11-AS promotes glioma cell growth and metastasis by targeting miR-130a-5p/HMGB2. Eur Rev Med Pharmacol Sci. 2019;23(1):241-52.

27. Zhang W, Zhang Y, Ding K, Zhang H, Zhao Q, Liu Z, et al. Involvement of JNK1/2-NF-kappaBp65 in the regulation of HMGB2 in myocardial ischemia/reperfusion-induced apoptosis in human AC16 cardiomyocytes. Biomed Pharmacother. 2018;106:1063-71.

28. Liu ZH, Dai DP, Ding FH, Pan WQ, Fang YH, Zhang Q, et al. Association of serum HMGB2 level with MACE at 1 mo of myocardial infarction: aggravation of myocardial ischemic injury in rats by HMGB2 via ROS. Am J Physiol Heart Circ Physiol. 2017;312(3):H422-36.

29. Tan XH, Zheng XM, Yu LX, He J, Zhu HM, Ge XP, et al. Fibroblast growth factor 2 protects against renal ischaemia/reperfusion injury by attenuating mitochondrial damage and proinflammatory signalling. J Cell Mol Med. 2017;21(11):2909-25.

30. Kocaturk H, Bedir F, Altay MS, Bakan E, Suleyman B, Yazici GN, Sunar $M$, Suleyman Z, Suleyman $H$. The effect of desloratadine on ischemia 
reperfusion induced oxidative and inflammatory renal injury in rats. Ren Fail. 2020:42(1):531-8.

31. Zhang W, Liu X, Jiang Y, Wang N, Li F, Xin H. 6-Gingerol attenuates ischemia-reperfusion-induced cell apoptosis in human AC16 cardiomyocytes through HMGB2-JNK1/2-NF-kappaB pathway. Evid Based Complement Alternat Med. 2019;2019:8798653.

32. He HY, Ren L, Guo T, Deng YH. Neuronal autophagy aggravates microglial inflammatory injury by downregulating CX3CL1/fractalkine after ischemic stroke. Neural Regen Res. 2019;14(2):280-8.
33. Lopes AP, van Roon JAG, Blokland SLM, Wang M, Chouri E, Hartgring SAY, et al. MicroRNA-130a contributes to type-2 classical DC-activation in Sjogren's syndrome by targeting mitogen- and stress-activated protein kinase-1. Front Immunol. 2019;10:1335.

\section{Publisher's Note}

Springer Nature remains neutral with regard to jurisdictional claims in published maps and institutional affiliations.
Ready to submit your research? Choose BMC and benefit from:

- fast, convenient online submission

- thorough peer review by experienced researchers in your field

- rapid publication on acceptance

- support for research data, including large and complex data types

- gold Open Access which fosters wider collaboration and increased citations

- maximum visibility for your research: over $100 \mathrm{M}$ website views per year

At BMC, research is always in progress.

Learn more biomedcentral.com/submissions 